\title{
Leadership in Singleton Congestion Games
}

\author{
Alberto Marchesi ${ }^{1}$, Stefano Coniglio ${ }^{2}$, Nicola Gatti ${ }^{1}$ \\ ${ }^{1}$ Politecnico di Milano, Piazza Leonardo da Vinci 32, Milano, Italy \\ ${ }^{2}$ University of Southampton, University Road SO17 1BJ, Southampton, United Kingdom \\ alberto.marchesi@polimi.it,s.coniglio@soton.ac.uk,nicola.gatti@polimi.it
}

\begin{abstract}
We study Stackelberg games where the underlying structure is a congestion game. We recall that, while leadership in 2-player games has been widely investigated, only few results are known when the number of players is three or more. The intractability of finding a Stackelberg equilibrium (SE) in normal-form and polymatrix games is among them. In this paper, we focus on congestion games in which each player can choose a single resource (a.k.a. singleton congestion games) and a player acts as leader. We show that, without further assumptions, finding an SE when the followers break ties in favor of the leader is not in Poly-APX, unless $P=N P$. Instead, under the assumption that every player has access to the same resources and that the cost functions are monotonic, we show that an SE can be computed efficiently when the followers break ties either in favor or against the leader.
\end{abstract}

\section{Introduction}

The problem of finding a Stackelberg equilibrium (SE) when mixed-strategy commitments are allowed is receiving a lot of attention in the artificial intelligence literature, also thanks to the many successful real-world applications in, e.g., security [Tambe, 2011]. In Stackelberg games, a player acts as leader, committing to a (potentially) mixed strategy, while the other players act as followers [Von Stengel and Zamir, 2010]. Different versions of SEs can be defined based on how the followers break ties: optimistic (OSE) if in favor of the leader, and pessimistic (PSE) if against her.

Finding an OSE or a PSE in 2-player normal-form games is easy, as shown by, respectively, Conitzer and Sandholm [2006] and Von Stengel and Zamir [2010]. The same holds for finding an OSE in $n$-player games where the followers play simultaneously in a correlated fashion [Conitzer and Korzhyk, 2011]. In more general situations, though, the problem is hard. Indeed, computing an O/PSE for normal-form games with two followers who play simultaneously a Nash equilibrium (NE) is not in Poly-APX unless P $=$ NP [Basilico et al., 2017]. In polymatrix games with the followers restricted to pure strategies, finding an OSE is not in Poly-APX unless $P=N P$ if the number of followers is not fixed, while it is easy if their number is a constant [De Nittis et al., 2018]. Finding a PSE is NP-hard in normal-form games even with two followers playing pure strategies [Coniglio et al., 2017]. Finding an OSE is also NP-hard [Conitzer and Sandholm, 2006] with multiple followers playing sequentially.

We focus, here, on congestion games (CGs) - game models which, in spite of their simplicity, enjoy nice computational properties even with many players-with the aim of investigating whether, in these games, a Stackelberg paradigm is computationally tractable. In CGs, given a set of resources, the players' actions are subsets of the resources and the costs the players perceive depend (monotonically or not) on the level of resource utilization (congestion). CGs always admit pure-strategy NEs which are achievable by best-response dynamics [Rosenthal, 1973; Monderer and Shapley, 1996]. CGs where each player cannot use more than a single resource are called singleton CGs (SCGs). Computing their NEs is easy [Ackermann et al., 2008]. Furthermore, in SCGs in which all the players have the same action space, finding a social-cost minimizing NE is also easy [Ieong et al., 2005].

Original contributions. We apply a Stackelberg paradigm to SCGs, assuming the presence of a special player acting as leader. We also allow the leader to perceive costs which are potentially different from the followers'. The leader commits to a (potentially) mixed strategy, while all the other players, acting as followers, observe the leader's commitment and then play, simultaneously, reaching an NE. ${ }^{1}$ In particular, we study the case in which the followers play pure strategies after observing the leader's commitment, which is reasonable as this followers' game always admits at least a purestrategy NE reachable by some best-response dynamics. A simple practical scenario is when a player has a higher priority to decide which resource to use before the other players, e.g., when the resources can be used for free, but gaining a higher priority requires a payment. We show that, when no further assumptions are made, computing an OSE is not in Poly-APX unless $P=N P$, even when the leader has only one available action and her costs are equal to the followers'. This shows that the same inapproximability result also holds

\footnotetext{
${ }^{1}$ To our knowledge, the only works related to ours are [Roughgarden, 2004; Fotakis, 2010] and their extensions. However, they analyze a different Stackelberg paradigm where the leader is an authority whose objective is to minimize the social cost of the NE reached by the followers.
} 
for finding an NE minimizing the cost for a given player in an SCG without leadership. Instead, when every player has access to the same set of resources and the costs are monotonically increasing functions of the congestion, the problem of finding an OSE or a PSE can be solved in polynomial time. Such result holds even when leader's and followers' costs are different. While the derivation is straightforward when the leader's commitment is a pure strategy, the analysis is more involved with mixed-strategy commitments, and the result follows from the fact that mixed-strategy commitments do not allow the leader to incur a cost smaller than the one she gets with pure-strategy ones. Furthermore, we show that the previous result holds only when the resource cost functions are monotonic, as, in the non-monotonic case, the leader's cost with mixed-strategy commitments can be strictly smaller than that obtained with a commitment in pure strategies.

\section{Preliminaries}

In this work, we analyze CGs in which a leader commits to a strategy beforehand, and, then, the followers simultaneously decide how to play, reaching an NE in the game that results from observing the leader's commitment. Following the notation by Shoham and Leyton-Brown (2008), we formally define a Stackelberg SCG (SSCG) as a tuple $\left(N, R, A, c_{\ell}, c_{f}\right)$ where: $N=F \cup\{\ell\}$ is a finite set of players, with player $\ell$ denoting the leader and $F$ the set of followers, $R$ is a finite set of resources, $A=\left\{A_{p}\right\}_{p \in N}$, where $A_{p} \subseteq R$ represents the set of player $p$ 's actions, and $c_{\ell}=\left\{c_{i, \ell}\right\}_{i \in R}$ and $c_{f}=\left\{c_{i, f}\right\}_{i \in R}$ are, respectively, the leader's and followers' cost functions, with $c_{i, \ell}, c_{i, f}: \mathbb{N} \rightarrow \mathbb{Q}$ denoting the costs of resource $i$ as a function of its congestion. As usual, we assume $c_{i, \ell}(0)=c_{i, f}(0)=0$ for every $i \in R$. In the following, let $|N|=n$ and $|R|=r$ be the number of players and resources, respectively.

A strategy $\sigma_{p}$ of player $p \in N$ is a probability distribution over $A_{p}$ where $\sigma_{p}\left(a_{p}\right)$ denotes the probability that $a_{p} \in A_{p}$ is played. Let $\Delta_{p}$ be the set of player $p$ 's strategies. A strategy $\sigma_{p} \in \Delta_{p}$ is said pure if it prescribes to always play action $a_{p} \in A_{p}$, i.e., $\sigma_{p}\left(a_{p}\right)=1$; otherwise, $\sigma_{p}$ is called mixed. A collection of players' strategies is called strategy profile in general, and action profile if all the strategies are pure. In this work, we use $\sigma=\left(\sigma_{\ell}, a\right)$ to collectively denote a strategy profile in which the leader plays a (potentially) mixed strategy $\sigma_{\ell} \in \Delta_{\ell}$ and the followers play pure strategies which determine an action profile $a=\left(a_{p}\right)_{p \in F} \in X_{p \in F} A_{p}$.

In the following, given a followers' action profile $a=$ $\left(a_{p}\right)_{p \in F} \in \times_{p \in F} A_{p}$, let $\nu_{i}^{a}=\left|\left\{p \in F \mid a_{p}=i\right\}\right|$ be the number of followers selecting resource $i \in R$ in $a$, i.e., the resource congestion caused by the followers' presence only. We define the followers' configuration induced by $a$ as the vector $\nu^{a} \in \mathbb{N}^{r}$ whose $i$-th component is $\nu_{i}^{a}$. In addition, for $\sigma_{\ell} \in \Delta_{\ell}$ we define $c_{i, f}^{\sigma_{\ell}}: \mathbb{N} \rightarrow \mathbb{Q}$, the followers' expected cost of resource $i \in R$ given $\sigma_{\ell}$, as a function of the number $x$ of followers selecting $i$, i.e., $c_{i, f}^{\sigma_{\ell}}(x)=\sigma_{\ell}(i) c_{i, f}(x+1)+\left(1-\sigma_{\ell}(i)\right) c_{i, f}(x)$. Indeed, given a leader's strategy $\sigma_{\ell}$, all followers who select resource $i \in R$ experience a congestion that may (with probability $\sigma_{\ell}(i)$ ) or may not (with probability $1-\sigma_{\ell}(i)$ ) be incre- mented by one, depending on whether the leader would or would not choose resource $i$. Finally, given $\sigma=\left(\sigma_{\ell}, a\right)$, let $c_{\ell}^{\sigma}=\sum_{i \in A_{\ell}} \sigma_{\ell}(i) c_{i, \ell}\left(\nu_{i}^{a}+1\right)$ be the leader's cost.

Notice that, after observing a leader's strategy $\sigma_{\ell}$, the followers play a new $\mathrm{CG}$ where resource costs are specified by functions $c_{i, f}^{\sigma_{\ell}}$, for $i \in R$. Being a CG, such game always admits an NE in which the players adopt pure strategies [Rosenthal, 1973]. Moreover, we assume that the followers play pure-strategy NEs, which are reached by playing some bestresponse dynamics [Monderer and Shapley, 1996].

Given strategy profile $\sigma=\left(\sigma_{\ell}, a\right), a$ is an NE for $\sigma_{\ell}$ if, for every $p \in F$ and $a_{p}^{\prime} \in A_{p}, c_{a_{p}, f}^{\sigma_{\ell}}\left(\nu_{a_{p}}^{a}\right) \leq c_{a_{p}^{\prime}, f}^{\sigma_{\ell}}\left(\nu_{a_{p}^{\prime}}^{a}+1\right)$, i.e., if no follower has an incentive to unilaterally deviate from $a_{p}$ by selecting another resource $a_{p}^{\prime}$. For $\sigma_{\ell} \in \Delta_{\ell}$, let $E^{\sigma_{\ell}}$ be the set of NEs in the followers' game resulting from $\sigma_{\ell}$.

In the second part of the work, we restrict our attention to a subclass of SSCGs where each player can select every resource, i.e., where $A_{p}=R$ for all $p \in N$. We refer to these games as Simple SSCGs (SSSCGs). Formally, an SSSCGs is a tuple $\left(N, R, c_{\ell}, c_{f}\right)$ whose elements are defined as in an SSCG where all the followers are identical as they are allowed to choose the same resources. Thus, only the number of followers selecting each resource is significant. As a consequence, a followers' action profile $a$ can be equivalently represented with the followers' configuration $\nu^{a}$ induced by it. Thus, when studying SSSCGs, we do not explicitly refer to followers' action profiles but, rather, use $\nu \in \mathbb{N}^{r}$ with $\sum_{i \in R} \nu_{i}=n-1$ to denote a followers' configuration. Moreover, let us notice that a followers' configuration $\nu$ is an NE for $\sigma_{\ell} \in \Delta_{\ell}$ if, for every $i \in R: \nu_{i}>0$ and $j \in R$, $c_{i, f}^{\sigma_{\ell}}\left(\nu_{i}\right) \leq c_{j, f}^{\sigma_{\ell}}\left(\nu_{j}+1\right)$.

Observe that, given a leader's strategy, there might be multiple NEs in the followers' game and, hence, different definitions of SE can be considered depending on the equilibriumselection rule adopted by the followers. As customary in the literature, we consider two definitions: optimistic SE (OSE) and pessimistic $S E$ (PSE). In the first one, the followers act in favor of the leader, thus selecting an NE minimizing her cost, while, in the second one, the followers always select an NE which results in the maximum leader's cost. Formally:

Definition 1. A strategy profile $\sigma=\left(\sigma_{\ell}, a\right)$ is an OSE if it solves the following bilevel problem:

$$
\min _{\sigma_{\ell} \in \Delta_{\ell}} \min _{a \in E^{\sigma_{\ell}}} c_{\ell}^{\left(\sigma_{\ell}, a\right)}
$$

As it is clear, an OSE always exists in SSCGs.

Definition 2. A PSE, if it exists, is a strategy profile $\sigma=$ $\left(\sigma_{\ell}, a\right)$ which solves the following bilevel problem:

$$
\min _{\sigma_{\ell} \in \Delta_{\ell}} \max _{a \in E^{\sigma_{\ell}}} c_{\ell}^{\left(\sigma_{\ell}, a\right)}
$$

Let us recall that, in general, the problem in Definition 2 may not admit a minimum (but only an infimum) and, thus, a PSE may not exist [Von Stengel and Zamir, 2010].

\section{SSCG NP-Hardness and Inapproximability}

Let us start our analysis with a negative result, showing that the problem of computing an OSE in SSCGs is computationally intractable, even if the leader can select a single resource 
and her costs are monotonic. Our result is based on a reduction from 3SAT, a well-known NP-complete problem [Garey and Johnson, 1979] which reads as follows:

Definition 3 (3SAT). Given a finite set $C$ of 3-literal clauses defined over a finite set $V$ of variables, is there a truth assignment to the variables which satisfies all clauses?

Theorem 1. Computing an OSE in SSCGs is NP-hard.

Proof. We provide a reduction from 3SAT showing that the existence of a polynomial-time algorithm for computing an OSE in SSCGs would allow us to solve any 3SAT instance in polynomial time. Specifically, given a $3 \mathrm{SAT}$ instance $(C, V)$ and a number $0<\epsilon<4$, we build an $\operatorname{SSCG~} \Gamma_{\epsilon}(C, V)$ such that there exists an OSE where the leader's cost is $\epsilon$ if and only if $(C, V)$ is satisfiable (if not, the leader's cost is 4 in any OSE). In the following, $l \in \phi$ denotes a literal (i.e., a variable or its negation) appearing in $\phi \in C$, while $v(l)$ denotes the variable corresponding to that literal. Moreover, we let $|C|=$ $m$ and $|V|=s$ be, respectively, the number of clauses and variables.

Mapping. $\Gamma_{\epsilon}(C, V)$ is defined as follows:

- $N=F \cup\{\ell\}$, with $F=\left\{p_{\phi}, p_{\phi, t} \mid \phi \in C\right\} \cup$ $\left\{p_{v, t}, p_{v}, p_{\bar{v}} \mid v \in V\right\} \cup\left\{p_{\phi, v}, p_{\phi, \bar{v}} \mid \phi \in C, v \in V\right\}$;

- $R=\left\{r_{t}\right\} \cup\left\{r_{\phi} \mid \phi \in C\right\} \cup\left\{r_{v, t}, r_{v}, r_{\bar{v}} \mid v \in V\right\} \cup$ $\left\{r_{\phi, v}, r_{\phi, \bar{v}} \mid \phi \in C, v \in V\right\}$;

- $A_{p_{\phi}}=\left\{r_{\phi}\right\} \cup\left\{r_{\phi, l} \mid l \in \phi\right\}, A_{p_{\phi, t}}=\left\{r_{\phi}, r_{t}\right\} \forall \phi \in C$;

- $A_{p_{v}}=\left\{r_{v, t}, r_{v}\right\}, A_{p_{\bar{v}}}=\left\{r_{v, t}, r_{\bar{v}}\right\}, A_{p_{v, t}}=$ $\left\{r_{v, t}, r_{t}\right\} \forall v \in V$

- $A_{p_{\phi, v}}=\left\{r_{v}, r_{\phi, v}\right\}, A_{p_{\phi, \bar{v}}}=\left\{r_{\bar{v}}, r_{\phi, \bar{v}}\right\} \quad \forall \phi \in C, v \in$ $V$

- $A_{\ell}=\left\{r_{t}\right\}$.

Moreover, cost functions are specified by the following table, where $c_{r_{\bar{v}}, f}=c_{r_{v}, f}, c_{r_{\phi, \bar{v}}, f}=c_{r_{\phi, v}, f}$, and $c_{r_{t}, f}=c_{r_{t}, \ell}$ :

\begin{tabular}{c|ccccc}
\hline$x$ & $c_{r_{\phi}, f}$ & $c_{r_{v}, f}$ & $c_{r_{v, t}, f}$ & $c_{r_{\phi, v}, f}$ & $c_{r_{t}, f}$ \\
\hline 1 & 2 & 7 & 7 & 1 & $\epsilon$ \\
{$[2, m]$} & 5 & 7 & 3 & 6 & 4 \\
$m+1$ & 5 & 0 & 3 & 6 & 4
\end{tabular}

Figure 1 shows an example of game $\Gamma_{\epsilon}(C, V)$. Clearly, given $(C, V), \Gamma_{\epsilon}(C, V)$ can be constructed in polynomial time, as it features $n=2 m+3 s+2 m s+1$ players and $r=m+3 s+2 m s+1$ resources. Observe that, in $\Gamma_{\epsilon}(C, V)$, the leader has a single resource available. Hence, the only leader's commitment is to select resource $r_{t}$, setting $\sigma_{\ell}\left(r_{t}\right)=1$. As a result, the leader's cost is $\epsilon$ if and only if no follower selects resource $r_{t}$; otherwise, it is 4 .

If. Suppose that $(C, V)$ is satisfiable, and let $\tau: V \rightarrow$ $\{\mathrm{T}, \mathrm{F}\}$ be a truth assignment satisfying all clauses in $C$. Using $\tau$, we recover a followers' action profile $a=\left(a_{p}\right)_{p \in F} \in$ $\times_{p \in F} A_{p}$ such that $a \in E^{\sigma_{\ell}}$, with $\sigma=\left(\sigma_{\ell}, a\right)$ providing the leader with a cost of $\epsilon$. Since $\epsilon$ is the minimum cost the leader can achieve and the followers behave optimistically, $\sigma$ is an OSE. In particular, let $a_{p_{\phi, t}}=r_{\phi}$, for all $\phi \in C$, and $a_{p_{v, t}}=r_{v, t}$, for all $v \in V$. Moreover, if $\tau(v)=\mathrm{T}$, let $a_{p_{v}}=a_{p_{\phi, v}}=r_{v}, a_{p_{\bar{v}}}=r_{v, t}$, and $a_{p_{\phi, \bar{v}}}=r_{\phi, \bar{v}}$ for all $\phi \in C$, while, if $\tau(v)=\mathrm{F}$, let $a_{p_{\bar{v}}}=a_{p_{\phi, \bar{v}}}=r_{\bar{v}}, a_{p_{v}}=r_{v, t}$, and $a_{p_{\phi, v}}=r_{\phi, v}$ for all $\phi \in C$. Notice that, since either
$\tau(v)=\mathrm{T}$ or $\tau(v)=\mathrm{F}$, one between $r_{v}$ and $r_{\bar{v}}$ is selected by $m+1$ followers and the other one by none, respectively. Say, w.l.o.g., $\nu_{r_{v}}^{a}=m+1$ and $\nu_{r_{\bar{v}}}^{a}=0$, as the other case is analogous. First, no follower $p_{\phi, v}$ would deviate from $r_{v}$ to $r_{\phi, v}$, as, otherwise, she would incur a cost of at least 1 , rather than 0 . The same holds for followers $p_{\phi, \bar{v}}$, as their cost is at most 6 while, if any of them switched to $r_{\bar{v}}$, she would incur a cost of 7. Similarly, since there are exactly two followers selecting $r_{v, t}$, follower $p_{v}$ would not deviate from $r_{v}$ (as $0<3$ ), while $p_{\bar{v}}$ and $p_{v, t}$ would not switch from $r_{v, t}$, as they would get 7 and 4 , respectively, rather than 3. Furthermore, since $\tau$ is a truth assignment satisfying $(C, V)$, at least one literal $l \in \phi$ evaluates to true under $\tau$ for every $\phi \in C$. Let $a_{p_{\phi}}=r_{\phi, l}$ for every $\phi \in C$. Since $l$ evaluates to true, it must be $a_{p_{\phi, l}}=r_{l}$, thus $p_{\phi}$ is the only follower who selects $r_{\phi, l}$. As a result, $p_{\phi}$ experiences a cost equal to 1 , and she has no incentive to deviate. Finally, $p_{\phi, t}$ does not deviate from $r_{\phi}$ to $r_{t}$ as $2<4$. Thus, we can conclude that $a$ is an NE and, since there is no follower using $r_{t}$, the leader's cost is $\epsilon$.

Only if. Suppose there exists an OSE $\sigma=\left(\sigma_{\ell}, a\right)$ in which the leader's cost is $\epsilon$. We show that $a=\left(a_{p}\right)_{p \in F} \in X_{p \in F} A_{p}$ can be employed to recover, in polynomial-time, a truth assignment $\tau$ that satisfies all clauses in $C$. First, let us note that no follower selects $r_{t}$ in $a$ as, otherwise, the leader's cost would be $4>\epsilon$. As a consequence, all followers $p_{\phi, t}$ and $p_{v, t}$ must select the other resource they have available, i.e, $a_{p_{\phi, t}}=r_{\phi}$ and $a_{p_{v, t}}=r_{v, t}$. Moreover, there cannot be two followers using resource $r_{\phi}$, for every $\phi \in C$, as, otherwise, $p_{\phi, t}$ would have an incentive to deviate from $r_{\phi}$ to $r_{t}$, as $5>4$. Thus, $a_{p_{\phi}} \neq r_{\phi}$, i.e., there must be a literal $l \in \phi$ such that $a_{p_{\phi}}=r_{\phi, l}$, for all $\phi \in C$. In addition, there cannot be two followers selecting $r_{\phi, l}$ as, otherwise, $p_{\phi}$ would have an incentive to deviate to $r_{\phi}$, as $5<6$. Thus, it must be the case that $a_{p_{\phi, l}}=r_{l}$. This implies that $\nu_{r_{l}}^{a}=m+1$ as, otherwise, the cost of $p_{\phi, l}$ would be $7>6$, and the follower would change resource, paying $r_{\phi, l}$. Furthermore, at least one between $p_{v}$ and $p_{\bar{v}}$ must select $r_{v, t}$ as, otherwise, player $p_{v, t}$ 's cost would be $7<4$, and she would prefer switching to resource $r_{t}$. As a result, at least one between $r_{v}$ and $r_{\bar{v}}$ must be selected by a number of followers strictly smaller than $m+1$; in that case, no follower $p_{\phi, v}$ (or $p_{\phi, \bar{v}}$ ) selects that resource as, otherwise, she would incur a cost of 7 and she would have an incentive to deviate. We thus define a truth assignment $\tau$ such that: $\tau(v)=\mathrm{T}$ if $\nu_{r_{v}}^{a}=m+1, \tau(v)=\mathrm{F}$ if $\nu_{r_{\bar{v}}}^{a}=m+1$, and $\tau(v)$ is either $\mathrm{T}$ or $\mathrm{F}$ whenever $\nu_{r_{v}}^{a}=\nu_{r_{\bar{v}}}^{a}=0$. Clearly, $\tau$ is well-defined. Moreover, we previously showed that, for every $\phi \in C$, there exists a literal $l \in \phi$ such that $a_{p_{\phi, l}}=r_{l}$, which implies that $r_{l}=m+1$, and, thus, $\tau(v(l))=\mathrm{T}$ if $l$ is positive, while $\tau(v(l))=\mathrm{F}$ if it is negative. Therefore, $\tau$ satisfies all clauses.

\section{The proof of Theorem 1 also shows the following:}

Observation 1. In SCGs without leadership, computing an NE minimizing the cost of a given player is NP-hard.

Furthermore, from Theorem 1, it directly follows that the leader's cost in an OSE cannot be efficiently approximated to within any approximation factor which depends polynomially on the size of the input: 


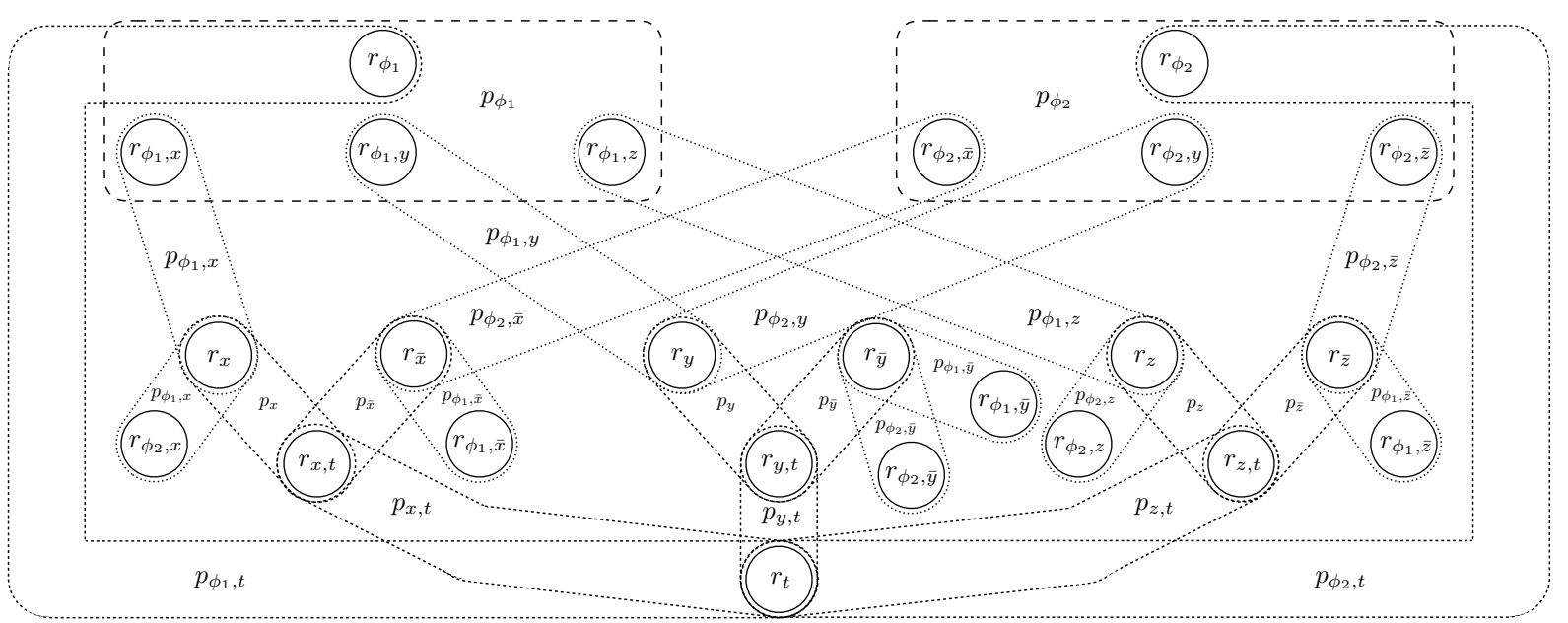

Figure 1: Example of $\Gamma_{\epsilon}(C, V)$ with $V=\{x, y, z\}$ and $C=\left\{\phi_{1}, \phi_{2}\right\}$, where $\phi_{1}=x \vee y \vee z$ and $\phi_{2}=\bar{x} \vee y \vee \bar{z}$.

Corollary 1. The problem of computing an OSE in SSCGs is not in Poly-APX unless $\mathrm{P}=\mathrm{NP}$.

Proof. Given a 3SAT instance $(C, V)$, let us build an SSCG $\Gamma_{\epsilon}(C, V)$ as in the proof of Theorem 1 . We have already proven that $\Gamma_{\epsilon}(C, V)$ has an OSE in which the leader's cost is $\epsilon$ if and only if $(C, V)$ is satisfiable and that, otherwise, the leader's cost is 4 . Let $\epsilon=\frac{4}{2^{n+r}}$. Assume that there exists a polynomial-time approximation algorithm $\mathcal{A}$ with approximation factor $\operatorname{poly}(n, r)$, i.e., a polynomial function of $n$ and $r$. Assume $(C, V)$ is satisfiable. $\mathcal{A}$ applied to $\Gamma_{\epsilon}(C, V)$ would return a solution with leader's cost at most $\frac{4}{2^{n+r}} \operatorname{poly}(n, r)$. Since, for $n$ and $r$ large enough, $\frac{4}{2^{n+r}} \operatorname{poly}(n, r)<4, \mathcal{A}$ would allow us to decide in polynomial time whether $(C, V)$ is satisfiable, a contradiction unless $P=N P$.

Notice that, since the followers break ties in favour of the leader in the reduction, the result in Theorem 1 does not apply to the problem of finding a PSE. Our conjecture is that, as it is the case for all the problems with known results on computing SEs, computing a PSE is as hard as finding an OSE.

\section{SSSCGs with Monotonic Costs}

We focus, in this section, on SSSCGs, showing that, assuming players' costs which are monotonic functions of the resource congestion, an O/PSE can be computed efficiently. Formally, we call the players' cost functions weakly monotonic if, for every resource $i \in R, c_{i, \ell}(x) \leq c_{i, \ell}(x+1)$ and $c_{i, f}(x) \leq$ $c_{i, f}(x+1)$ for all $x \in \mathbb{N}$, and strictly monotonic if all the inequalities are strict.

First, notice that, in these games, searching for an O/PSE is not as easy as it might appear, for the following reason:

Observation 2. There are SSSCGs with weakly monotonic cost functions where some followers' configurations are NEs only for leader's mixed strategies.

Consider a game with three followers, $R=\left\{r_{1}, r_{2}, r_{3}\right\}$, and followers' costs as in Figure 2(a). The followers' configuration in which each follower selects a different resource is not an NE if the leader commits to a pure strategy, while, for instance, it is an NE for $\sigma_{\ell}\left(r_{1}\right)=\sigma_{\ell}\left(r_{3}\right)=\frac{1}{2}, \sigma_{\ell}\left(r_{2}\right)=0$.

In the following, we show that, when searching for an OSE, one can restrict the attention without loss of generality to pure strategies of the leader, provided that the players' cost functions are weakly monotonic.

Intuitively, given an OSE in which the leader plays a mixed strategy, we can easily construct another equilibrium in which, instead, the leader's strategy is pure.

Theorem 2. Every SSSCG with weakly monotonic cost functions admits an OSE $\sigma=\left(\sigma_{\ell}, \nu\right)$ in which $\sigma_{\ell}$ is pure.

Proof. Given an OSE $\sigma=\left(\sigma_{\ell}, \nu\right)$, with $\sigma_{\ell}$ mixed, we construct another OSE $\hat{\sigma}=\left(\hat{\sigma}_{\ell}, \hat{\nu}\right)$ such that $\hat{\sigma}_{\ell}$ is pure. Let $S=\left\{i \in R \mid \sigma_{\ell}(i)>0\right\}$ be the set of resources played by the leader with positive probability in $\sigma_{\ell}$, and let $i^{\star} \in$ $\arg \min _{i \in S} c_{i, \ell}\left(\nu_{i}+1\right)$. Clearly, $c_{\ell}^{\sigma}=\sum_{i \in A_{\ell}} \sigma_{\ell}(i) c_{i, \ell}\left(\nu_{i}+\right.$ 1) $\geq c_{i^{\star}, \ell}\left(\nu_{i^{\star}}+1\right)$. Moreover, given that $\nu$ is an NE for $\sigma_{\ell}$, the following holds:

$$
c_{i, f}^{\sigma_{\ell}}\left(\nu_{i}\right) \leq c_{j, f}^{\sigma_{\ell}}\left(\nu_{j}+1\right) \forall i \in R: \nu_{i}>0, j \in R .
$$

Let us define $\hat{\sigma}_{\ell}$ such that $\hat{\sigma}_{\ell}\left(i^{\star}\right)=1$. We now show that such $\hat{\sigma}_{\ell}$ is part of an OSE. Notice that $c_{i, f}^{\hat{\sigma}_{\ell}}(x)=c_{i, f}(x) \forall x \in \mathbb{N}$ for every $i \neq i^{\star} \in R$, while $c_{i^{\star}, f}^{\hat{\sigma}_{\ell}}(x)=c_{i^{\star}, f}(x+1) \forall x \in \mathbb{N}$. Given that the followers behave optimistically, it is sufficient to provide a $\hat{\nu} \in E^{\hat{\sigma}_{\ell}}$ such that $\hat{\sigma}=\left(\hat{\sigma}_{\ell}, \hat{\nu}\right)$ satisfies $c_{\ell}^{\hat{\sigma}} \leq c_{\ell}^{\sigma}$. Specifically, we construct a sequence of followers' configurations reaching such $\hat{\nu}$. Given $\hat{\sigma}_{\ell}$, let us consider the sequence $(\nu(0)=\nu, \nu(1), \ldots, \nu(T)=\hat{\nu})$ such that each configuration differs from the previous one in that a single follower has changed resource, strictly decreasing her cost in the followers' game resulting from $\hat{\sigma}_{\ell}$. Formally, for all $0 \leq t<T$, there exists $i, j \in R$ such that $\nu(t)_{i}>0, \nu(t+1)_{i}=\nu(t)_{i}-1$, $\nu(t+1)_{j}=\nu(t)_{j}+1$, and $c_{i, f}^{\hat{\sigma}_{\ell}}\left(\nu(t)_{i}\right)>c_{j, f}^{\hat{\sigma}_{\ell}}\left(\nu(t+1)_{j}\right)$. Moreover, let us assume that a follower deviates to resource $i^{\star}$, i.e., $\nu(t+1)_{i^{\star}}>\nu(t)_{i^{\star}}$, only if this is the only way of 
Proceedings of the Twenty-Seventh International Joint Conference on Artificial Intelligence (IJCAI-18)

strictly decreasing some follower's cost. Now, we prove:

$$
\nu(t+1)_{i^{\star}} \leq \nu(t)_{i^{\star}} \forall 0 \leq t<T .
$$

By contradiction, suppose there exists $0 \leq t<T$ such that $\nu(t+1)_{i^{\star}}>\nu(t)_{i^{\star}}$. Then, in $\nu(t)$, there exists a follower who can strictly decrease her cost by choosing $i^{\star}$ instead of resource $j \neq i^{\star} \in R: \nu(t)_{j}>0$. Thus,

$$
c_{i^{\star}, f}^{\sigma_{\ell}}\left(\nu_{i^{\star}}+1\right) \leq c_{i^{\star}, f}\left(\nu(t)_{i^{\star}}+2\right)<c_{j, f}\left(\nu(t)_{j}\right),
$$

where the first inequality holds since $\nu(t)_{i^{\star}}=\nu_{i^{\star}}$. Two cases are possible. In the first one, $\nu(t)_{j} \leq \nu_{j}$, implying $c_{j, f}\left(\nu(t)_{j}\right) \leq c_{j, f}\left(\nu_{j}\right) \leq c_{j, f}^{\sigma_{\ell}}\left(\nu_{j}\right)$, which, together with Equations (1) and (3), leads to a contradiction. In the second case, $\nu(t)_{j}>\nu_{j}$ implies that there exists $k \neq i^{\star} \in R$ such that $\nu(t)_{k}<\nu_{k}$ (and $\nu_{k}>0$ ), otherwise $\sum_{i \in R} \nu(t)_{i}>n-1$. It follows that $c_{j, f}\left(\nu(t)_{j}\right) \leq c_{k, f}\left(\nu(t)_{k}+1\right) \leq c_{k, f}^{\sigma_{\ell}}\left(\nu_{k}\right)$, where the first inequality holds since, due to our assumptions on the sequence, it cannot be $c_{j, f}\left(\nu(t)_{j}\right)>c_{k, f}\left(\nu(t)_{k}+1\right)$ as $\nu(t+1)_{i^{\star}}>\nu(t)_{i^{\star}}$, and the second inequality follows from $\nu(t)_{k}<\nu_{k}$. Thus, Equations (1) and (3) give a contradiction. As a result, Equation (2) holds, and, thus, $\hat{\nu}_{i^{\star}} \leq \nu_{i^{\star}}$. Given the monotonicity of the costs, $\hat{\sigma}$ is an OSE.

Now, we prove that a similar result holds for the pessimistic case. The result is weaker though, as it requires the stronger assumption that the followers' cost functions be strictly monotonic.

Theorem 3. Every SSSCG in which leader's and followers' cost functions are weakly monotonic and strictly monotonic, respectively, admits a PSE $\sigma=\left(\sigma_{\ell}, \nu\right)$ in which $\sigma_{\ell}$ is pure.

Proof. Suppose there exists a PSE $\sigma=\left(\sigma_{\ell}, \nu\right)$ in which $\sigma_{\ell}$ is mixed. We show that there must be another PSE $\hat{\sigma}=\left(\hat{\sigma}_{\ell}, \hat{\nu}\right)$ such that $\hat{\sigma}_{\ell}$ is pure. Let us define $i^{\star} \in R$ as in the proof of Theorem 2, so that $c_{\ell}^{\sigma} \geq c_{i^{\star}, \ell}\left(\nu_{i^{\star}}+1\right)$ and Equation (1) holds. Given that the followers behave pessimistically, we need to show that, for every $\hat{\nu} \in E^{\hat{\sigma}_{\ell}}, \hat{\sigma}=\left(\hat{\sigma}_{\ell}, \hat{\nu}\right)$ satisfies $c_{\ell}^{\hat{\sigma}} \leq c_{\ell}^{\sigma}$. By contradiction, assume that $c_{\ell}^{\hat{\sigma}}>c_{\ell}^{\sigma}$, which implies $c_{i^{\star}, \ell}\left(\hat{\nu}_{i^{\star}}+1\right)>c_{i^{\star}, \ell}\left(\nu_{i^{\star}}+1\right)$. It easily follows from the monotonicity of the costs that $\hat{\nu}_{i^{\star}}>\nu_{i^{\star}}$. Thus, there must be a resource $j \in R$ such that $\hat{\nu}_{j}<\nu_{j}$, otherwise $\sum_{i \in R} \hat{\nu}_{i}>n-1$. Moreover, let us notice that $\nu_{j}>0$. Thus,

$c_{i^{\star}, f}^{\sigma_{\ell}}\left(\nu_{i^{\star}}+1\right) \leq c_{i^{\star}, f}\left(\hat{\nu}_{i^{\star}}+1\right) \leq c_{j, f}\left(\hat{\nu}_{j}+1\right) \leq c_{j, f}^{\sigma_{\ell}}\left(\nu_{j}\right)$,

where the first inequality follows from $\nu_{i^{\star}}<\hat{\nu}_{i^{\star}}$, the second one from the fact that $\hat{\nu}$ is an NE for $\hat{\sigma}_{\ell}$, while the third one from $\hat{\nu}_{j}<\nu_{j}$. Equation (1) implies $c_{j, f}^{\sigma_{\ell}}\left(\nu_{j}\right) \leq c_{i^{\star}, f}^{\sigma_{\ell}}\left(\nu_{i^{\star}}+1\right)$. If $c_{j, f}^{\sigma_{\ell}}\left(\nu_{j}\right)<c_{i^{\star}, f}^{\sigma_{\ell}}\left(\nu_{i^{\star}}+1\right)$, then Equation (4) leads to a contradiction. Otherwise, if $c_{j, f}^{\sigma_{\ell}}\left(\nu_{j}\right)=c_{i_{\ell}, f}^{\sigma_{\ell}}\left(\nu_{i^{\star}}+1\right)$, all inequalities in Equation (4) must hold as equalities. However, this would imply $c_{i^{\star}, f}^{\sigma_{\ell}}\left(\nu_{i^{\star}}+1\right)=c_{i^{\star}, f}\left(\hat{\nu}_{i^{\star}}+1\right)$ and $c_{j, f}\left(\hat{\nu}_{j}+1\right)=c_{j, f}^{\sigma_{\ell}}\left(\nu_{j}\right)$, a contradiction as $\sigma_{\ell}$ is mixed and the followers' cost functions are strictly monotonic.

Moreover, let us notice that Theorem 3 fails to hold whenever the followers' cost functions are weakly monotonic.

\begin{tabular}{c|ccc}
\hline$x$ & $c_{r_{1}, f}$ & $c_{r_{2}, f}$ & $c_{r_{3}, f}$ \\
\hline 1 & 1 & 4 & 0 \\
2 & 3 & 7 & 2 \\
3 & 5 & 7 & 5
\end{tabular}

(a)

\begin{tabular}{c|cc|cc}
\hline$x$ & $c_{r_{1}, \ell}$ & $c_{r_{1}, f}$ & $c_{r_{2}, \ell}$ & $c_{r_{2}, f}$ \\
\hline 1 & 1 & 2 & 1 & 2 \\
2 & 2 & 1 & 2 & 1
\end{tabular}

(c)

\begin{tabular}{c|cc|cc}
\hline$x$ & $c_{r_{1}, \ell}$ & $c_{r_{1}, f}$ & $c_{r_{2}, \ell}$ & $c_{r_{2}, f}$ \\
\hline 1 & 1 & 1 & 1 & 1 \\
2 & 2 & 1 & 2 & 1
\end{tabular}

(b)

\begin{tabular}{c|cc|cc}
\hline$x$ & $c_{r_{1}, \ell}$ & $c_{r_{1}, f}$ & $c_{r_{2}, \ell}$ & $c_{r_{2}, f}$ \\
\hline 1 & 2 & 1 & 2 & 1 \\
2 & 0 & 2 & 0 & 2
\end{tabular}

(d)
Figure 2: Cost functions of some SSSCG examples.

Observation 3. There are SSSCGs with weakly monotonic cost functions where any PSE prescribes the leader to play a mixed strategy.

Consider a game with two followers, $R=\left\{r_{1}, r_{2}\right\}$, and players' costs as in Figure 2(b). Clearly, any followers' configuration is an NE, independently of the leader's commitment. Thus, whenever the leader commits to a pure strategy, she incurs a cost of 2 , while she can pay only 1 by uniformly randomizing between the two resources.

Theorems 2 and 3 provide the fundamental insights which allow us to efficiently compute O/PSEs in SSSCGs with monotonic cost functions. Specifically, we can compute an OSE (resp., PSE) by enumerating the leader's pure strategies and, for each of them, computing the followers' NE which results in the leader's cost being minimized (resp., maximized). An O/PSE is then obtained by picking a pure strategy which minimizes then leader's cost. The detailed procedure is described in Algorithm 1, where function $\mathrm{O}-\mathrm{Pick}(S)$ (resp., $\mathrm{P}-\mathrm{Pick}(S)$ ) returns some resource $j^{\star} \in S$, giving precedence to resources $j^{\star} \neq i$ (resp., $j^{\star}=i$ ).

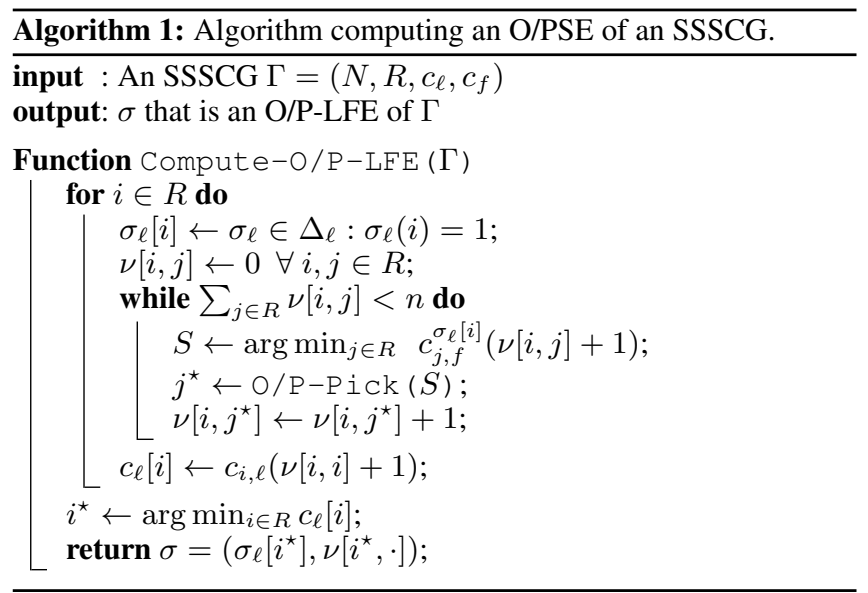

Let us remark that, in Algorithm $1, \sigma_{\ell}[\cdot], \nu[\cdot, \cdot]$, and $c_{\ell}[\cdot]$ are the algorithm's variables, and, for every $i \in R, \nu[i, j]$ denotes the number of followers selecting resource $j \in R$ in the NE that is reached when the leader's strategy is $\sigma_{\ell}[i]$.

Theorem 4. Algorithm 1 is correct and it runs in time $O(n r \log r)$. 
Proof. In order to show that Algorithm 1 is correct, due to Theorems 2 and 3 we only need to prove that, for every $i \in R$ and after the execution of the while loop, the followers' configuration $\nu$ is such that $\nu_{j}=\nu[i, j]$ for $j \in R$ is an NE for $\sigma_{\ell}[i]$ minimizing (or maximizing) the leader's cost. First, let us show that $\nu$ is an NE. Suppose, by contradiction, it is not. Then, there exists $j \in R: \nu_{j}>0$ and $k \in R$ such that $c_{j, f}^{\sigma_{\ell}[i]}\left(\nu_{j}\right)>c_{k, f}^{\sigma_{\ell}[i]}\left(\nu_{k}+1\right)$. Let $\bar{\nu}_{k}$ be the value of $\nu[i, k]$ during the step in which $\nu[i, j]$ is set to its final value $\nu_{j}$. Clearly, $c_{j, f}^{\sigma_{\ell}[i]}\left(\nu_{j}\right)>c_{k, f}^{\sigma_{\ell}[i]}\left(\nu_{k}+1\right) \geq c_{k, f}^{\sigma_{\ell}[i]}\left(\bar{\nu}_{k}+1\right)$, and the algorithm would have not incremented $\nu[i, j]$ during that step, a contradiction. In the rest of the proof, we focus on the optimistic case, as the pessimistic one can be treated analogously. Suppose, by contradiction, that $\nu$ is not an NE minimizing the leader's cost for $\sigma_{\ell}[i]$. Then, there exists another NE $\hat{\nu}$ for $\sigma_{\ell}[i]$ such that $c_{i, \ell}\left(\hat{\nu}_{i}+1\right)<c_{i, \ell}\left(\nu_{i}+1\right)$. Given the monotonicity of the costs, $\hat{\nu}_{i}<\nu_{i}$. Therefore, there must exist $j \neq i \in R$ such that $\hat{\nu}_{j}>\nu_{j}$. Let us consider the step in which $\nu[i, i]$ is set to $\nu_{i}$, and let $\bar{\nu}_{j}$ be the value of $\nu[i, j]$ during that step. It must be that $c_{i, f}^{\sigma_{\ell}[i]}\left(\nu_{i}\right)<c_{j, f}^{\sigma_{\ell}[i]}\left(\bar{\nu}_{j}+1\right)$ as, otherwise, the algorithm would have incremented $\nu[i, j]$ instead of $\nu[i, i]$. But then $c_{j, f}^{\sigma_{\ell}[i]}\left(\bar{\nu}_{j}+1\right) \leq c_{j, f}^{\sigma_{\ell}[i]}\left(\nu_{j}+1\right) \leq c_{j, f}^{\sigma_{\ell}[i]}\left(\hat{\nu}_{j}\right)$, which implies $c_{i, f}^{\sigma_{\ell}[i]}\left(\hat{\nu}_{i}+1\right) \leq c_{i, f}^{\sigma_{\ell}[i]}\left(\nu_{i}\right)<c_{j, f}^{\sigma_{\ell}[i]}\left(\bar{\nu}_{j}+1\right) \leq$ $c_{j, f}^{\sigma_{\ell}[i]}\left(\hat{\nu}_{j}\right)$, contradicting the fact that $\hat{\nu}$ is an NE for $\sigma_{\ell}[i]$.

Clearly, the while loop is executed exactly $r$ times, and each execution performs $n$ steps. Moreover, using efficient data structures each step takes time $O(\log r)$. Thus, the overall running time is $O(n r \log r)$

We conclude the section by showing that, in SSSCGs with monotonic costs and under the additional assumption that leader's and followers' costs be equal, all O/PSEs in which the leader plays a pure strategy are NEs in the game where all players play simultaneously (i.e., without leadership).

Theorem 5. Given an SSSCG with monotonic costs and $c_{\ell}=$ $c_{f}=\left\{c_{i}\right\}_{i \in R}$, any O/PSE $\sigma=\left(\sigma_{\ell}, a\right)$ with $\sigma_{\ell}$ pure is an NE.

Proof. Let $\sigma=\left(\sigma_{\ell}, \nu\right)$ be an O/PSE with $\sigma_{\ell}\left(i^{\star}\right)=1$ for some $i^{\star} \in R$. Clearly, given that $\nu \in E^{\sigma_{\ell}}$, for every $i \in R$ : $\nu_{i}>0$ and $j \in R, c_{i}^{\sigma_{\ell}}\left(\nu_{i}\right) \leq c_{j}^{\sigma_{\ell}}\left(\nu_{j}+1\right)$. Therefore, there is no follower who has an incentive to change resource, and, thus, it is sufficient to prove that the leader does not deviate from resource $i^{\star}$ either, unilaterally. If $\nu_{i^{\star}}>0$, we have $c_{i^{\star}}\left(\nu_{i^{\star}}+1\right)=c_{i^{\star}}^{\sigma_{\ell}}\left(\nu_{i^{\star}}\right) \leq c_{j}^{\sigma_{\ell}}\left(\nu_{j}+1\right)=c_{j}\left(\nu_{j}+1\right)$ for every $j \neq i^{\star} \in R$, and it immediately follows that the leader does not deviate and $\sigma$ is an NE. The case in which $\nu_{i^{\star}}=0$ is more involved. By contradiction, suppose that $\sigma$ is not an NE. As a consequence, the leader must have an incentive to deviate for some $j \neq i^{\star} \in R$, i.e., $c_{i^{\star}}\left(\nu_{i^{\star}}+1\right)=c_{i^{\star}}(1)>c_{j}\left(\nu_{j}+1\right)$. Suppose the leader commits to a strategy $\hat{\sigma}_{\ell}$ such that $\hat{\sigma}_{\ell}(j)=$ 1. We prove that, for every $\hat{\nu} \in E^{\hat{\sigma}_{\ell}}, \hat{\sigma}=\left(\hat{\sigma}_{\ell}, \hat{\nu}\right)$ provides the leader with a cost strictly smaller than $c_{i^{\star}}(1)$. Suppose, instead, $c_{j}\left(\hat{\nu}_{j}+1\right) \geq c_{i^{\star}}(1)$. Three cases are possible. In the first case, $\hat{\nu}_{j}<\nu_{j}$ and $c_{i^{\star}}(1)>c_{j}\left(\nu_{j}+1\right) \geq c_{j}\left(\hat{\nu}_{j}+\right.$ $1) \geq c_{i^{\star}}(1)$. In the second one, $\hat{\nu}_{j}=\nu_{j}$ and $c_{j}\left(\hat{\nu}_{j}+1\right) \geq$ $c_{i^{\star}}(1)>c_{j}\left(\nu_{j}+1\right)$. Finally, in the third case, $\hat{\nu}_{j}>\nu_{j}$, which implies that there must be a resource $k \neq i^{\star} \in R$ such that $\hat{\nu}_{k}<\nu_{k}$, and $c_{i^{\star}}(1)>c_{j}\left(\nu_{j}+1\right) \geq c_{k}\left(\nu_{k}\right) \geq c_{k}\left(\hat{\nu}_{k}+1\right) \geq$ $c_{j}\left(\hat{\nu}_{j}+1\right) \geq c_{i^{\star}}(1)$. As all cases lead to a contradiction, it must be $c_{j}\left(\hat{\nu}_{j}+1\right)<c_{i^{\star}}(1)$. The proof is complete as, in $\hat{\sigma}$, the leader's cost is $c_{j}\left(\hat{\nu}_{j}+1\right)<c_{i^{\star}}(1)$, contradicting the fact that $\sigma$ is an O/PSE.

\section{SSSCGs with Arbitrary Costs}

Finally, let us shift our attention to general SSSCGs, i.e., games in which the costs need not be monotonic functions of the resource congestion.

Observation 4. Given an SSSCG, an optimal leader's pure strategy to commit to can be computed efficiently, both in the optimistic and the pessimistic case.

Clearly, when the followers' costs are monotonic functions, we can find an optimal leader's pure strategy using Algorithm 1. In general, we can apply a procedure similar to that of Algorithm 1, enumerating the leader's pure strategies while computing, for each of them, an NE minimizing/maximizing the leader's cost in the resulting followers' game. In order to find one such NE, we can adapt an algorithm proposed in [Ieong et al., 2005], which relies on dynamic programming to compute in $O\left(r^{5} n^{6}\right)$ an NE minimizing the social-cost in SSSCGs without leadership. It suffices to change the objective function from the social cost to the leader's cost of the resource selected in the current pure strategy. Thus, in general, the overall computation requires $O\left(r^{6} n^{6}\right)$.

Unfortunately, the assumption that the leader always plays pure strategies is not safe in SSSCGs with arbitrary costs, as Theorems 2 and 3 do not hold if the monotonicity assumption is dropped.

Observation 5. There are SSSCGs such that:

- the followers' costs only are non-monotonic, and any O/PSE prescribes the leader to play a mixed strategy;

- the leader's costs only are non-monotonic, and any O/PSE prescribes the leader to play a mixed strategy.

Consider a game with a single follower, $R=\left\{r_{1}, r_{2}\right\}$, and players' costs as in Figure 2(c). Clearly, the follower selects $r_{2}$ whenever $\sigma_{\ell}\left(r_{1}\right) \leq \frac{1}{2}$, while, if $\sigma_{\ell}\left(r_{1}\right) \geq \frac{1}{2}$, she chooses $r_{1}$, providing the leader with a cost of $2-\sigma_{\ell}\left(r_{1}\right)$ and $1+\sigma_{\ell}\left(r_{1}\right)$, respectively. Thus, any O/PSE prescribes the leader to play $\sigma_{\ell}$ such that $\sigma_{\ell}\left(r_{1}\right)=\frac{1}{2}$.

Moreover, when the players' costs are as in Figure 2(d), the follower selects $r_{2}$ if $\sigma_{\ell}\left(r_{1}\right) \geq \frac{1}{2}$, and $r_{1}$ if $\sigma_{\ell}\left(r_{1}\right) \leq \frac{1}{2}$, providing the leader with a cost of $2 \sigma_{\ell}\left(r_{1}\right)$ and $2-2 \sigma_{\ell}\left(r_{1}\right)$, respectively. As a result, any O/PSE of the game prescribes the leader to play $\sigma_{\ell}$ such that $\sigma_{\ell}\left(r_{1}\right)=\frac{1}{2}$.

\section{Conclusions and Future Works}

We analyzed Stackelberg games where the underlying structure is a congestion game, focusing on the case in which the players' actions are singletons. We proved that, without further assumptions on the players' action spaces and the resource cost functions, it is not possible to approximate in polynomial time the leader's cost in an OSE up to within a polynomial factor in the size of the game, unless $P=N P$. 
Differently, when each player can select any resource and the cost functions are monotonic, an O/PSE can be computed efficiently, as there is always a leader's optimal pure strategy.

In the future, we will study the computational complexity of finding an O/PSE in SSSCGs with arbitrary costs in order to establish whether the problem can be solved efficiently or not, in spite of the fact that the leader's optimal commitment may be a mixed strategy. Moreover, we will extend our results for SSCGs - studying the complexity of finding a PSE, also considering the special case where cost functions are monotonic in the resource congestion-and we will analyze other classes of CGs with different combinatorial structures.

\section{References}

[Ackermann et al., 2008] Heiner Ackermann, Heiko Röglin, and Berthold Vöcking. On the impact of combinatorial structure on congestion games. Journal of the ACM (JACM), 55(6):25, 2008.

[Basilico et al., 2017] Nicola Basilico, Stefano Coniglio, and Nicola Gatti. Methods for finding leader-follower equilibria with multiple followers. CoRR, abs/1707.02174, 2017.

[Coniglio et al., 2017] Stefano Coniglio, Nicola Gatti, and Alberto Marchesi. Pessimistic leader-follower equilibria with multiple followers. In IJCAI, pages 171-177, 2017.

[Conitzer and Korzhyk, 2011] Vincent Conitzer and Dmytro Korzhyk. Commitment to correlated strategies. In $A A A I$, pages 632-637, 2011.

[Conitzer and Sandholm, 2006] Vincent Conitzer and Tuomas Sandholm. Computing the optimal strategy to commit to. In Proceedings of the 7th ACM conference on Electronic commerce, pages 82-90, 2006.

[De Nittis et al., 2018] Giuseppe De Nittis, Alberto Marchesi, and Nicola Gatti. Computing the optimal strategy to commit to in polymatrix games. In $A A A I$, pages 82-90, 2018.

[Fotakis, 2010] Dimitris Fotakis. Stackelberg strategies for atomic congestion games. Theory of Computing Systems, 47(1):218-249, 2010.

[Garey and Johnson, 1979] M. R Garey and D. S. Johnson. Computers and Intractability: A Guide to the Theory of NP-completeness. WH Freeman and Company, 1979.

[Ieong et al., 2005] Samuel Ieong, Robert McGrew, Eugene Nudelman, Yoav Shoham, and Qixiang Sun. Fast and compact: A simple class of congestion games. In $A A A I$, pages 489-494, 2005.

[Monderer and Shapley, 1996] Dov Monderer and Lloyd S Shapley. Potential games. Games and economic behavior, 14(1):124-143, 1996.

[Rosenthal, 1973] Robert W Rosenthal. A class of games possessing pure-strategy nash equilibria. International Journal of Game Theory, 2(1):65-67, 1973.

[Roughgarden, 2004] Tim Roughgarden. Stackelberg scheduling strategies. SIAM Journal on Computing, 33(2):332-350, 2004.
[Shoham and Leyton-Brown, 2008] Yoav Shoham and Kevin Leyton-Brown. Multiagent systems: Algorithmic, game-theoretic, and logical foundations. Cambridge University Press, 2008.

[Tambe, 2011] Milind Tambe. Security and Game Theory: Algorithms, Deployed Systems, Lessons Learned. Cambridge University Press, 2011.

[Von Stengel and Zamir, 2010] Bernhard Von Stengel and Shmuel Zamir. Leadership games with convex strategy sets. Games and Economic Behavior, 69(2):446-457, 2010 . 